\title{
Solving a Delay Differential Equation by Two Direct
}

\section{Approaches}

\author{
N. O. Alatawi and A. Ebaid \\ Department of Mathematics, Faculty of Science, University of Tabuk, P.O. Box 741, Tabuk 71491, Saudi Arabia
}

\begin{abstract}
The Ambartsumian equation is an application of the theory of surface brightness in the milky way. It is a linear differential equation involving a proportional delay term. In this paper, two different approaches have been used to obtain the power series solution of Ambartsumian equation. The obtained series solution by the suggested approaches agrees with the published solution in the literature. However, the current approaches are not only very simple but also straightforward.
\end{abstract}

Key words: Ambartsumian equation, milky way, series solution.

\section{Introduction}

In celestial mechanics, the Ambartsumian equation is used in the theory of surface brightness in the milky way [1]. It is a linear differential equation involving a proportional delay term, and given by Ref. [2]

$$
y^{\prime}(t)=-y(t)+\frac{1}{q} y\left(\frac{t}{q}\right), q>1
$$

where $q$ is a constant for the given model and Eq. (1) is subjected to the initial condition:

$$
y(0)=\lambda
$$

where $\lambda$ is also a constant. Existence and uniqueness have been proved and discussed by Kato and McLeod [3]. Searching for simple analytical solution for the Ambartsumian equation is still of manifest practical interest. In order to contribute to an improved solution of this problem, the authors believe that the series methods are effective in dealing with this equation. The objective of this paper is to analyze the Ambartsumian equation by using two different methods based on the power series solution. We show that the two methods lead to the same solution which

Corresponding author: Abdelhalim Ebaid, $\mathrm{PhD}$, associate professor, research fields: applied differential equations with applications in physics and biomathematics, nanofluids, special relativity. agrees with that one in the literature.

\section{First Method}

In this section, we search for a solution of Eq. (1) in the following form:

$$
y=\sum_{n=0}^{\infty} a_{n}(q) t^{n} .
$$

On inserting Eq. (3) into Eq. (1), we have

$$
\sum_{n=0}^{\infty} n a_{n}(q) t^{n-1}=-\sum_{n=0}^{\infty} a_{n}(q) t^{n}+\frac{1}{q} \sum_{n=0}^{\infty} a_{n}(q) \frac{t^{n}}{q^{n}},(4)
$$

or

$$
\sum_{n=1}^{\infty} n a_{n}(q) t^{n-1}=\sum_{n=0}^{\infty}\left[\frac{1}{q^{n+1}}-1\right] a_{n}(q) t^{n} .
$$

Collecting the like terms of same powers, yields

$\sum_{n=0}^{\infty}\left[(n+1) a_{n+1}(q)-\left(\frac{1}{q^{n+1}}-1\right) a_{n}(q)\right] t^{n}=0$.

Therefore

$$
a_{n+1}(q)=\left[\frac{q^{-(n+1)}-1}{n+1}\right] a_{n}(q), \quad \mathrm{n} \geq 0 .
$$

Accordingly, 


$$
\begin{aligned}
& a_{1}=\frac{1}{1 !}\left(q^{-1}-1\right) a_{0}, \\
& a_{2}=\frac{1}{2 !}\left(q^{-1}-1\right)\left(q^{-2}-1\right) a_{0}=\frac{1}{2 !} \prod_{k=1}^{2}\left(q^{-k}-1\right) a_{0}, \\
& a_{3}=\frac{1}{3 !}\left(q^{-1}-1\right)\left(q^{-2}-1\right)\left(q^{-3}-1\right) a_{0}=\frac{1}{3 !} \prod_{k=1}^{3}\left(q^{-k}-1\right) a_{0}, \\
& a_{4}=\frac{1}{4 !}\left(q^{-1}-1\right)\left(q^{-2}-1\right)\left(q^{-3}-1\right)\left(q^{-4}-1\right) a_{0}=\frac{1}{4 !} \prod_{k=1}^{4}\left(q^{-k}-1\right) a_{0}, \\
& \cdot \\
& a_{n}=\frac{1}{n !}\left(q^{-1}-1\right)\left(q^{-2}-1\right) \ldots\left(q^{-(n-1)}-1\right)\left(q^{-n}-1\right) a_{0}=\frac{1}{n !} \prod_{k=1}^{n}\left(q^{-k}-1\right) a_{0} .
\end{aligned}
$$

Hence

$$
\begin{aligned}
y(t) \quad & =a_{0}+\sum_{n=1}^{\infty} a_{n} t^{n} \\
& =a_{0}+\sum_{n=1}^{\infty} \frac{1}{n !} \prod_{k=1}^{n}\left(q^{-k}-1\right) a_{0} t^{n}, \\
& =a_{0}\left[1+\sum_{n=1}^{\infty}\left(\prod_{k=1}^{n}\left(q^{-k}-1\right)\right) \frac{t^{n}}{n !}\right] .
\end{aligned}
$$

On using the initial condition in Eq. (2), we finally obtain

$$
y=\lambda\left[1+\sum_{n=1}^{\infty}\left(\prod_{k=1}^{n}\left(q^{-k}-1\right)\right) \frac{t^{n}}{n !}\right] .
$$

\section{Second Method}

In this section, we show the applicability of the Maclaurin expansion:

$$
y=\sum_{n=0}^{\infty} \frac{y^{(n)}(0)}{n !} t^{n}
$$

for the delay differential Eq. (1). To do that, we first obtain from Eq. (1) that:

$$
y^{(1)}(0)=-y(0)+\frac{1}{q} y(0)=\lambda\left(q^{-1}-1\right) .
$$

Then, we differentiate Eq. (12) once again with respect to $t$ to obtain:

$$
y^{(2)}(t)=-y^{(1)}(t)+\frac{1}{q^{2}} y^{(1)}\left(\frac{t}{q}\right),
$$

and hence,

$$
\begin{gathered}
y^{(2)}(0)=\left(q^{-2}-1\right) y^{(1)}(0)= \\
\lambda\left(q^{-1}-1\right)\left(q^{-2}-1\right) .
\end{gathered}
$$

Differentiation of Eq. (13) once again with respect to $t$ gives:

$$
y^{(3)}(t)=-y^{(2)}(t)+\frac{1}{q^{3}} y^{(2)}\left(\frac{t}{q}\right) .
$$

Accordingly,

$$
\begin{gathered}
y^{(3)}(0)=\left(q^{-3}-1\right) y^{(2)}(0)= \\
\lambda\left(q^{-1}-1\right)\left(q^{-2}-1\right)\left(q^{-3}-1\right) .
\end{gathered}
$$

Repeating this procedure $n$-times, we get 


$$
\begin{aligned}
y^{(n)}(0) & =\lambda\left(q^{-1}-1\right)\left(q^{-2}-1\right) \ldots \ldots\left(q^{-(n-1)}-1\right)\left(q^{-n}-1\right), \\
& =\lambda \prod_{k=1}^{n}\left(q^{-k}-1\right), \quad n \geq 1 .
\end{aligned}
$$

Therefore

$$
\begin{aligned}
y(t) & =y(0)+\sum_{n=1}^{\infty} \frac{y^{(n)}(0)}{n !} t^{n}, \\
& =\lambda+\sum_{n=1}^{\infty} \frac{\lambda}{n !} \prod_{k=1}^{n}\left(q^{-k}-1\right) t^{n}, \\
& =\lambda\left[1+\sum_{n=1}^{\infty}\left(\prod_{k=1}^{n}\left(q^{-k}-1\right)\right) \frac{t^{n}}{n !}\right],
\end{aligned}
$$

which is also the same closed form solution obtained in Ref. [2] by using Daftardar-Gejji and Jafari Method [4].

\section{Conclusions}

In this paper, a delay differential equation arising in Astrophysics has been analytically solved. Two different series approaches have been applied on the Ambartsumian equation. It was shown that the obtained solution agrees with the series solution in the literature. In conclusion, the present approaches can be extended to deal with a generalized form of this equation.

\section{References}

[1] Ambartsumian, V. A. 1994. "On the Fluctuation of the Brightness of the Milky Way." Doklady Akad Nauk USSR 44: 223-6.

[2] Patade, J., and Bhalekar, S. 2017. "On Analytical Solution of Ambartsumian Equation." Natl. Acad. Sci. Lett. doi: 10.1007/s40009-017-0565-2.

[3] Kato, T., and McLeod, J. B. 1971. "The Functional-Differential Equation $y^{\prime}(x)=a y(\lambda x)+b y(x)$." Bull. Am. Math. Soc. 77: 891-935.

[4] Daftardar-Gejji, V., and Bhalekar, S. 2008. "Solving Fractional Diffusion-Wave Equations Using the New Iterative Method." Fract. Calc. Appl. Anal. 11: 193-202. 